St udy of el ectromagnet i c mi croi nst abi l i ti es i n hel i cal systems wi th the stel I ar at or expansi on met hod

\begin{tabular}{|l|l|}
\hline $\begin{array}{l}\text { jour nal or } \\
\text { publ i cat i on } \mathrm{titl} \text { e }\end{array}$ & Physi cs of Pl asmas \\
\hline vol une & Vol . 11 \\
\hline number & I ssue 6 \\
\hline page range & pp. 3068- 3077 \\
\hline year & 2004 06-01 \\
\hline URL & ht t p: //hdl . handl e. net /10655/3835 \\
\hline
\end{tabular}




\title{
Study of electromagnetic microinstabilities in helical systems with the stellarator expansion method
}

\author{
H. Sugama and T.-H. Watanabe \\ National Institute for Fusion Science, Graduate University for Advanced Studies, Toki 509-5292, Japan
}

(Received 22 January 2004; accepted 8 March 2004; published online 6 May 2004)

\begin{abstract}
Electromagnetic microinstabilities in helical systems are studied by numerically solving integral eigenmode equations, which are derived from the ion gyrokinetic equation, the quasineutrality equation, the Ampère's law, and the massless electron approximation. The stellarator expansion technique is used to evaluate finite-beta effects on the guiding-center drift in the helical configuration, where the toroidal plasma shift and the magnetic shear strongly influence the magnetic curvature and accordingly the stability of both magnetohydrodynamics (MHD) and kinetic modes. The kinetic integral equations are shown to reduce to the ideal MHD ballooning mode equation in the fluid limit, from which the Mercier criterion is obtained. For helical geometry like the Large Helical Device (LHD) [Motojima, et al., Nucl. Fusion 43, 1674 (2003)], it is confirmed that, when increasing the beta value, the ion temperature gradient mode is stabilized while the kinetic ballooning mode $(\mathrm{KBM})$ is destabilized due to the unfavorable geodesic curvature resulting from the negative magnetic shear combined with the toroidal plasma shift. Also, dependencies of these kinetic-mode properties on the poloidal wave number and the magnetic shear are investigated. It is found that the KBM-unstable parameter region is narrower than the Mercier-unstable region in the LHD-like configuration. (C) 2004 American Institute of Physics. [DOI: 10.1063/1.1730294]
\end{abstract}

\section{INTRODUCTION}

Microinstabilities in magnetically confined plasmas have been studied extensively as a key mechanism for producing plasma turbulence and resultant anomalous transport. ${ }^{1}$ Above all, ion temperature gradient (ITG) modes and turbulence driven by them have been most actively investigated by numerous theories and simulations in recent years. ${ }^{2}$ The ITG mode is essentially an electrostatic instability, which is more unstable for lower-beta plasmas, and electromagnetic microinstabilities such as the kinetic ballooning mode $(\mathrm{KBM})^{3}$ are anticipated to become an active source of turbulence and transport in high-beta regimes. For the ITG mode, electrons are often assumed to adiabatically respond to electrostatic fluctuations while, in the electromagnetic case, it is necessary to include more complicated nonadiabatic electron dynamics due to magnetic fluctuations. So far, for helical systems, theoretical studies of microinstabilities have also been concerned with electrostatic modes mainly ${ }^{4-6}$ and those of electromagnetic modes have not been done sufficiently compared with tokamak cases. Since, recently, helical systems such as the Large Helical Device (LHD) ${ }^{7}$ have succeeded in producing high-beta plasmas, understanding the physical mechanism of their anomalous transport requires electromagnetic-microinstability analyses. Also, electromagnetic microinstabilities are deeply related to the ideal magnetohydrodynamics (MHD) interchange and ballooning modes with short wavelengths. In the LHD, a large pressure gradient is observed even in the Mercier-unstable region. ${ }^{7,8}$ Therefore, it is interesting to examine how the stability criterion based on the ideal MHD is modified by the microinstability analysis, which takes account of kinetic effects such as the Landau damping and the finite gyroradii.
In the present work, we investigate electromagnetic microinstabilities in helical systems with the LHD-like magnetic configuration. Recently, the KBM in the LHD was also studied by Yamagishi et al. ${ }^{9}$ using the ordinary differential eigenmode equation derived by Tang et al., ${ }^{3}$ in which the poloidal wavelength is assumed to be much larger than the ion thermal gyroradius. Here, in order to fully take account of the finite-gyroradius effect on the electromagnetic mode, we use the kinetic integral eigenmode equations similar to those by Dong et al. for tokamaks,${ }^{10}$ which are derived from the ion gyrokinetic equation, ${ }^{11,12}$ the quasineutrality condition, Ampère's law, and the massless electron approximation. Our numerical solution to the kinetic integral eigenmode equations utilizes procedures by Sugama ${ }^{13}$ for proper analytic continuation of the dispersion relation in the complex frequency plane, by which we can calculate both positive and negative growth rates so as to accurately determine the critical condition for the marginal stability.

Helical ripples and safety-factor profiles in the LHD-like configuration present a striking contrast to those in tokamaks. The ideal MHD ballooning mode in tokamaks is stable for the negative magnetic shear $d q / d r<0$ ( $q$ : the safety factor, $r$ : the minor radius) while, as shown by Nakajima, ${ }^{14}$ the unfavorable geodesic curvature resulting from the negative shear combined with a toroidal (Shafranov) shift of the finite-beta helical plasma destabilizes the ballooning mode in the LHD configuration. Here, we employ the stellarator expansion method ${ }^{15}$ to evaluate the finite-beta toroidal shift, which critically affects local magnetic shear, magnetic curvature, guiding-center drift, and resultantly stability of both MHD and kinetic modes. In fact, Nakamura et al. ${ }^{16}$ showed that the stellarator expansion well describes beta dependence 
of the magnetic axis position and the magnetic well depth in the heliotron-type configuration by comparison with results from three-dimensional equilibrium calculations. The kinetic eigenmode equations are shown to reduce to the ideal MHD ballooning equation in the fluid limit, from which the Mercier criterion for the helical plasma is obtained. Then, we can make a comparison between kinetic results from the microinstability analysis and the Mercier criterion.

The rest of this paper is organized as follows. In Sec. II, equilibria of helical plasmas are treated by the stellarator expansion technique to derive useful formulas for evaluation of the toroidal plasma shift and the magnetic drift for the finite-beta case. The kinetic integral eigenmode equations for electromagnetic microinstabilities are derived in Sec. III A and the ideal MHD ballooning equation is obtained from them in the fluid limit in Sec III. B, where we also derive the Mercier criterion which takes account of the finite-beta helical configuration by using the formulas in Sec. II. In Sec. IV, the kinetic integral eigenmode equations are numerically solved to investigate dependencies of electromagnetic-mode properties on the plasma beta, the magnetic shear, and the poloidal wave number. There, the relation of the kinetic results to the Mercier parameter is also examined. Finally, conclusions are given in Sec. V.

\section{EQUILIBRIA OF HELICAL PLASMAS}

Here, we use the toroidal coordinates $(r, \theta, \zeta)$, where $r$, $\theta$, and $\zeta$ denote the minor radius, the poloidal angle, and the toroidal angle, respectively, and they are related to the conventional cylindrical coordinates $(R, \phi, Z)$ with

$$
R=R_{0}+\Delta(r)+r \cos \theta, \quad \phi=-\zeta, \quad Z=r \sin \theta .
$$

Here the point defined by $R=R_{0}$ and $Z=0$ represents the geometrical center of the poloidal cross section of the wall boundary (or the external helical coils), and $\Delta(r)$ is defined later.

In the stellarator expansion, ${ }^{15}$ the lowest-order poloidal flux function $\psi \equiv-\left(4 \pi^{2} R_{0}\right)^{-1} \int \mathbf{B} \cdot \nabla \theta d V$ is independent of $\zeta$, and is written as $\psi=A+\psi_{h}$. Here $A$ is the toroidal $(\zeta)$ component of the magnetic vector potential associated with the plasma current and $\psi_{h}$ represents the contribution from the external helical fields. The function $A$ is determined by

$$
\nabla_{\perp}^{2} A=-4 \pi \Omega \frac{d p}{d \psi}+G(\psi),
$$

where $\nabla_{\perp}^{2}$ denotes the two-dimensional Laplacian in the plane perpendicular to the toroidal direction, the equilibrium pressure $p(\psi)$ and $G(\psi)$ are flux functions, and $\Omega$ is given by

$$
\Omega=\frac{2\left(R-R_{0}\right)}{R_{0}}+\Omega_{h} .
$$

Here, $\Omega_{h}$ is associated with the contribution of the helical field to the averaged magnetic curvature. Hereafter, we assume that there is no net toroidal current and that $\psi_{h}$ $=\psi_{h}\left(r_{0}\right)$ and $\Omega_{h}=\Omega_{h}\left(r_{0}\right)$ are functions of $r_{0} \equiv\left[\left(R-R_{0}\right)^{2}\right.$ $\left.+Z^{2}\right]^{1 / 2} \simeq r+\Delta(r) \cos \theta$ only, where $r_{0}$ is the minor radius measured from the center (or magnetic axis) of the vacuum helical field. Now, we take $\psi=\psi(r)$ so that the poloidal cross section labeled by $\psi$ is a circle with the radius $r$, the center of which is shifted from $R=R_{0}$ by $\Delta(r)(\ll r)$. Neglecting small terms $\sim(\Delta / r)^{2}$, Eq. (2) is rewritten as

$$
\begin{aligned}
\nabla_{\perp}^{2}[ & \left.\psi(r)-\psi_{h}(r)-\Delta(r) \psi_{h}^{\prime}(r) \cos \theta\right] \\
& \simeq-4 \pi \frac{p^{\prime}(r)}{\psi^{\prime}(r)}\left[\frac{2 r}{R_{0}} \cos \theta+\Omega_{h}(r)\right]+G(r),
\end{aligned}
$$

where ${ }^{\prime} \equiv d / d r$ and the pressure gradient $p^{\prime}$ is regarded as a small quantity of $\mathcal{O}(\Delta / r)$. We obtain $G(r)$ $=4 \pi \Omega_{h}(r) p^{\prime}(r) / \psi^{\prime}(r)$ from the no net toroidal current condition, $\left\langle\nabla_{\perp}^{2} A\right\rangle=0$, where $\langle\cdot\rangle$ denotes the flux surface average. Then, separating Eq. (4) into the $\theta$-averaged part and the $\theta$-dependent part, we find $\psi(r)=\psi_{h}(r)$ and

$$
\left(\Delta \psi^{\prime}\right)^{\prime \prime}+\frac{\left(\Delta \psi^{\prime}\right)^{\prime}}{r}-\frac{\Delta \psi^{\prime}}{r^{2}}=4 \pi \frac{p^{\prime}}{\psi^{\prime}} \frac{2 r}{R_{0}},
$$

respectively. Defining the safety factor $q(r)$ $\equiv-r B_{0} /\left(R_{0} \psi^{\prime}(r)\right)$ and the local plasma beta value $\beta(r)$ $\equiv 8 \pi p(r) / B_{0}^{2}$, and using Eq. (5) yield

$$
\Delta(r)=R_{0} q(r) \int_{0}^{r} \frac{d x}{x^{3}} \int_{0}^{x} y^{2} q(y) \beta^{\prime}(y) d y+\frac{q(r) \Delta(0)}{q(0)},
$$

where the toroidal shift $\Delta(0)$ of the magnetic axis can experimentally be controlled by the external vertical field. [Equation (6) is equivalent to Eq. (7.112) in Freidberg. ${ }^{17}$ ] From Eq. (6), we can show

$$
\Delta^{\prime}(r)=\frac{R_{0} q(r)}{r^{3}} \int_{0}^{r} x^{2} q(x) \beta^{\prime}(x) d x+\hat{s}(r) \frac{\Delta(r)}{r},
$$

and

$$
\begin{aligned}
r \Delta^{\prime \prime}(r)= & -\alpha(r)+[2 \hat{s}(r)-3] \Delta^{\prime}(r) \\
& +\left[2 \hat{s}(r)-\hat{s}^{2}(r)+r \hat{s}^{\prime}(r)\right] \frac{\Delta(r)}{r},
\end{aligned}
$$

where the magnetic shear parameter $\hat{s}(r) \equiv r q^{\prime}(r) / q(r)$ and $\alpha(r) \equiv-R_{0} q^{2}(r) \beta^{\prime}(r)$ are defined. The toroidal force balance for the finite-beta case causes the toroidal axis shift and the poloidal field compression, which are taken into account by Eqs. (6)-(8). In the well-known $s$ - $\alpha$ model, $\left|\Delta^{\prime \prime}\right|$ $\gg\left|\Delta^{\prime}\right| / r \sim|\Delta| / r^{2}$ is used and only the first term $-\alpha$ on the right-hand side of Eq. (8) is retained by assuming that the pressure profile has a steep gradient only around the surface considered (see Sec. 10.5.5 in Freidberg ${ }^{17}$ ). This term is responsible for the second stabilization of the ballooning mode in high beta regimes. However, it was pointed out by Nakajima $^{14}$ following the high beta model by Coppi et al. ${ }^{18}$ that, in the helical system, the second group of terms $(2 \hat{s}(r)-3) \Delta^{\prime}(r)$ on the right-hand side should also be kept and combined with the geodesic curvature in order to explain the destabilization mechanism of the ballooning mode for the case of the negative magnetic shear $\hat{s}<0$. Using the expres- 
sion for $\Delta^{\prime \prime}$ in Eq. (8), we can naturally unify the conventional $s$ - $\alpha$ model based on the assumption of the steep pressure gradient and the high beta model by Coppi et al. ${ }^{18}$ and Nakajima ${ }^{14}$ who used the near-axis expansion.

In the ballooning representation, ${ }^{19,20}$ we treat the fluctuation which varies rapidly across the field lines and has the wave number vector perpendicular to $\mathbf{b} \equiv \mathbf{B} / B$ denoted by

$$
\mathbf{k}_{\perp}=k_{n} \frac{\boldsymbol{\nabla} r}{|\boldsymbol{\nabla} r|}+k_{t} \frac{\mathbf{b} \times \boldsymbol{\nabla} r}{|\boldsymbol{\nabla} r|} .
$$

In the present case, the component $k_{t}$ tangential to the flux surface is approximately equal to the poloidal wave number, $k_{t} \simeq k_{\theta}=n q(r) / r$, where $n$ is the toroidal mode number. The ratio $\Sigma$ of the normal component $k_{n}$ to the tangential one $k_{t}$ is written as

$$
\Sigma \equiv \frac{k_{n}}{k_{t}} \simeq \hat{s}\left(\theta-\theta_{k}\right)+\left[\Delta^{\prime}(1+\hat{s})+r \Delta^{\prime \prime}\right] \sin \theta,
$$

where $\mathcal{O}\left(\Delta^{\prime}\right)$ terms are neglected except for the term $\Delta^{\prime}(1$ $+\hat{s}) \sin \theta$ that significantly affects the ballooning mode stability. From Eqs. (9) and (10), we have

$$
k_{\perp}^{2}=k_{\theta}^{2}\left[1+\Sigma^{2}\right]
$$

When evaluating the guiding-center drift which is responsible for ballooning-type instabilities, we take into account effects of helical ripples in the magnetic field strength by using $B=B_{0}\left[1-\epsilon_{00}(r)-\epsilon_{t}(r) \cos \theta-\epsilon_{h}(r) \cos (L \theta-M \zeta)\right]$, where $L$ and $M$ are the poloidal and toroidal period numbers of the helical fields, respectively ( $L=2$ and $M=10$ for the LHD). Here, $\epsilon_{t}=r / R_{0}$ and $\epsilon_{h}(r)\left(\propto r^{L}\right)$ represent the toroidicity and helicity parameters, respectively. The stellarator expansion technique is used to give $\epsilon_{00}(r)=\frac{1}{2}[\langle\Omega\rangle+\beta(r)]$, which is associated with the averaged normal magnetic curvature and the diamagnetic effect. Then, the magnetic drift frequency for the particle species $a$ with the mass $m_{a}$ and the charge $e_{a}$ is given by

$$
\omega_{D}=2 \epsilon_{n} \omega_{* a}\left(v_{\|}^{2}+v_{\perp}^{2} / 2\right) v_{T a}^{-2} G_{c}(\theta),
$$

where $\epsilon_{n}=L_{n} / R_{0}$ is the ratio of the density gradient scale length $L_{n} \equiv-\left(d \ln n_{0} / d \ln r\right)^{-1}$ to the major radius $R_{0}, \omega_{* a}$ $\equiv-k_{\theta} c T_{a} /\left(e_{a} L_{n}\right)$ is the diamagnetic frequency, $v_{\|}\left(v_{\perp}\right)$ is the parallel (perpendicular) velocity, and $v_{T a} \equiv\left(2 T_{a} / m_{a}\right)^{1 / 2}$ is the thermal velocity. Also, the dimensionless curvature factor $G_{c}(\theta)$ is defined along the field line labeled with $\alpha_{0}$ $=\zeta-q \theta$ by

$$
\begin{aligned}
G_{c}(\theta) \equiv & R_{0}[(\mathbf{b} \cdot \nabla) \mathbf{b} \times \mathbf{b}] \cdot\left(\mathbf{k}_{\perp} / k_{t}\right) \\
= & R_{0}\langle\Omega\rangle^{\prime} / 2+\cos \theta+L\left(\epsilon_{h} / \epsilon_{t}\right) \cos \{(L-M q) \theta \\
& \left.-M \alpha_{0}\right\}+\Sigma(\theta)\left[\sin \theta+L\left(\epsilon_{h} / \epsilon_{t}\right) \sin \{(L-M q) \theta\right. \\
& \left.\left.-M \alpha_{0}\right\}\right] .
\end{aligned}
$$

Unfavorable (favorable) curvature is indicated by $G_{c}>0$ $(<0)$. On the right-hand side of Eq. (13), the first term is derived from the averaged normal curvature, the second and third terms correspond to the normal curvature due to the toroidicity and the helicity, while the fourth and fifth terms with the coefficient $\Sigma$ contain effects of the toroidal and he- lical geodesic curvature, respectively. From Eqs. (1) and (3), the averaged normal curvature term $\langle\Omega\rangle^{\prime}$ is written as

$$
\langle\Omega\rangle^{\prime}=\Omega_{h}^{\prime}(r)+\frac{2}{R_{0}}\langle\Delta+r \cos \theta\rangle^{\prime} .
$$

Here, from Ref. 21, the averaged helical curvature is expressed in terms of $q$ and $\hat{s}$ as

$$
\Omega_{h}^{\prime}(r)=\frac{M}{L} \frac{\left(r^{4} / q\right)^{\prime}}{R_{0}^{2} r^{2}}=\frac{M}{L} \frac{r}{R_{0}^{2} q}(4-\hat{s}) .
$$

The contribution of the toroidal plasma shift to the normal curvature is represented by

$$
\begin{aligned}
\frac{2}{R_{0}}\langle\Delta+r \cos \theta\rangle^{\prime}= & \frac{\left[\left(r^{2} \Delta\right)^{\prime} / r\right]^{\prime}}{R_{0}} \\
= & \frac{1}{R_{0}}\left[-\alpha(r)+2 \hat{s} \Delta^{\prime}\right. \\
& \left.+\left(2 \hat{s}-\hat{s}^{2}+r \hat{s}^{\prime}\right) \frac{\Delta}{r}\right],
\end{aligned}
$$

where Eqs. (6) $-(8)$ and $\langle\cdot\rangle \simeq \oint \cdot\left(1+\Delta^{\prime} \cos \theta\right) d \theta /(2 \pi)$ are used. We see from Eqs. (8), (10), (13), (14), and (16) that $\alpha>0$ included in $\Delta^{\prime \prime}$ reduces $G_{c}$ and accordingly contributes to stabilization by entering both the averaged normal curvature term $\langle\Omega\rangle^{\prime}$ and the $\Sigma$ term combined with the geodesic curvature. In helical systems with negative magnetic shear $\hat{s}<0$, destabilization occurs from an increase in $G_{c}$ due to $\hat{s} \Delta^{\prime}>0$ that also appears in both $\langle\Omega\rangle^{\prime}$ and $\Sigma$.

In Sec. III, the gyrokinetic analysis of electromagnetic microinstabilities in helical plasmas is carried out based on the geometrical expressions shown in this section.

\section{EIGENMODE EQUATIONS}

Here, the kinetic integral eigenmode equations are derived and the ideal MHD ballooning equation is obtained from them in the fluid limit.

\section{A. Kinetic integral eigenmode equations}

The distribution function in the $(\mathbf{x}, \mathbf{v})$ phase space for the species $a=i$ (ion) or $e$ (electron) is divided into the equilibrium and perturbation parts as $f_{a}=n_{0} F_{\mathrm{M} a}+\delta f_{a}$ where $n_{0}$ is the equilibrium density and $F_{\mathrm{M} a} \equiv \pi^{-3 / 2} v_{T a}^{-3} \exp \left(-v^{2} / v_{T a}^{2}\right)$ is the Maxwellian distribution function. In the magnetic field $\mathbf{B}$, the perturbation part $\delta f_{a}$ with the perpendicular wave number vector $\mathbf{k}_{\perp}$ is written as

$$
\delta f_{a}=-\frac{e_{a} \phi}{T_{a}} n_{0} F_{\mathrm{M} a}+h_{a} e^{-i \mathbf{k}_{\perp} \cdot \boldsymbol{\rho}_{a}},
$$

where $\phi$ represents the electrostatic potential, $\boldsymbol{\rho}_{a} \equiv \mathbf{b} \times \mathbf{v} / \Omega_{a}$ denotes the gyroradius vector, and $\Omega_{a} \equiv e_{a} B /\left(m_{a} c\right)$ is the gyrofrequency. Here, the first and second terms on the righthand side of Eq. (17) represent the adiabatic and nonadiabatic parts, respectively. The nonadiabatic distribution function $h_{a}$ is independent of the gyrophase and is described in the collisionless linear electromagnetic case by the gyrokinetic equation, ${ }^{11,12}$ 


$$
\begin{aligned}
& {\left[\frac{v_{\|}}{R_{0} q} \frac{\partial}{\partial \theta}-i\left(\omega-\omega_{D a}\right)\right] h_{a}} \\
& \quad=-i\left(\omega-\omega_{* T a}\right) n_{0} F_{\mathrm{M} a} J_{0}\left(k_{\perp} \rho_{a}\right) \frac{e_{a}}{T_{a}}\left(\phi-\frac{v_{\|}}{c} A_{\|}\right),
\end{aligned}
$$

where $A_{\|}=\delta \mathbf{A} \cdot \mathbf{b}$ is the parallel component of the vector potential for the magnetic field perturbation, $\omega_{D a}$ is given by Eq. (12), and

$$
\omega_{* T a}=\omega_{* a}\left[1+\eta_{a}\left\{\left(\frac{v}{v_{T a}}\right)^{2}-\frac{3}{2}\right\}\right] .
$$

Here, $J_{0}$ is the Bessel function of order zero and $\eta_{a}$ $\equiv d \ln T_{a} / d \ln n_{0}$ is the ratio of the temperature gradient to the density gradient. In Eq. (18), we have neglected the parallel magnetic field perturbation and used the ballooning representation to regard the poloidal angle $\theta$ as a coordinate along the magnetic field line which forms the so-called covering space $(-\infty<\theta<+\infty) .{ }^{19,20}$

Throughout this work, following Dong et al., ${ }^{10}$ effects of magnetic geometry in helical systems are considered only through the magnetic drift and the perpendicular wave number vector described in the previous section. Trapped particle effects and variation in the parallel velocity $v_{\|}$along the field line are neglected because such instabilities as the ITG mode and the ballooning mode treated here are driven by passing particles mainly. Then, the solution of Eq. (18) with the boundary conditions $\lim _{\theta \rightarrow \pm \infty} h_{a}(\theta)=0$ is given by

$$
h_{a}= \begin{cases}-i \int_{-\infty}^{\theta} d \theta^{\prime}\left(R_{0} q /\left|v_{\|}\right|\right) e^{i \zeta_{a}\left(\theta, \theta^{\prime}\right)}\left(\omega-\omega_{* T a}\right) n_{0} F_{\mathrm{M} a} J_{0}\left(k_{\perp}^{\prime} \rho_{a}\right) \frac{e_{a}}{T_{a}}\left(\phi-\frac{v_{\|}}{c} A_{\|}\right) & \text {for } v_{\|}>0 \\ -i \int_{\theta}^{+\infty} d \theta^{\prime}\left(R_{0} q /\left|v_{\|}\right|\right) e^{-i \zeta_{a}\left(\theta, \theta^{\prime}\right)}\left(\omega-\omega_{* T a}\right) n_{0} F_{\mathrm{M} a} J_{0}\left(k_{\perp}^{\prime} \rho_{a}\right) \frac{e_{a}}{T_{a}}\left(\phi-\frac{v_{\|}}{c} A_{\|}\right) & \text {for } v_{\|}<0,\end{cases}
$$

where $k_{\perp}^{\prime}$ is given by Eq. (32) and

$$
\zeta_{a}\left(\theta, \theta^{\prime}\right)=\int_{\theta^{\prime}}^{\theta} d \theta^{\prime \prime}\left(R_{0} q /\left|v_{\|}\right|\right)\left[\omega-\omega_{D a}\left(\theta^{\prime \prime}\right)\right]
$$

In the ballooning representation, the boundary conditions $\lim _{\theta \rightarrow \pm \infty} \phi(\theta)=\lim _{\theta \rightarrow \pm \infty} A_{\|}(\theta)=0$ are also employed. Using the solution given by Eq. (20), we can calculate the density perturbation, $\delta n_{a} \equiv \int d^{3} v \delta f_{a}=-n_{0} e_{a} \phi / T_{a}+\int d^{3} v$ $\times J_{0}\left(k_{\perp} \rho_{a}\right) h_{a}$, and the parallel current perturbation, $\delta J_{\|}$ $\equiv \Sigma_{a=i, e} e_{a} \int d^{3} v J_{0}\left(k_{\perp} \rho_{a}\right) h_{a} v_{\|}$.

In the same way as in Dong et al. ${ }^{10}$ we here take the limit of the small electron-ion mass ratio, $m_{e} / m_{i} \rightarrow+0$. Then, we see that, in Eq. (18) for electrons $(a=e)$, the terms proportional to the parallel velocity $\left(v_{\|} \sim v_{T e}\right)$ are dominant and their balance yields the constraint for the lowest order of $A_{\|}$in the $\left(m_{e} / m_{i}\right)^{1 / 2}$-expansion,

$$
\int_{-\infty}^{+\infty} d k A_{\|}(k)=0
$$

where $k \equiv\left|\hat{s} k_{\theta}\right|\left(\theta-\theta_{k}\right)$ is used as an independent variable instead of $\theta$. Equation (22) implies that the electric field integrated along the field line $\int E_{\|} d l=i(\omega / c) \int A_{\|} d l$ should vanish in order to forbid unlimited acceleration of massless electrons. The quasineutrality condition $\Sigma_{a=i, e} e_{a} \delta n_{a}=0$ is rewritten as

$$
\begin{aligned}
\left(1+\tau_{e}\right) \phi(k)= & \int_{-\infty}^{+\infty} \frac{d k^{\prime}}{\sqrt{2 \pi}}\left[K_{11}^{i}\left(k, k^{\prime}\right) \phi\left(k^{\prime}\right)\right. \\
& \left.+\left\{K_{12}^{i}\left(k, k^{\prime}\right)+K_{12}^{e}\left(k, k^{\prime}\right)\right\} \frac{v_{T i}}{c} A_{\|}\left(k^{\prime}\right)\right],
\end{aligned}
$$

where $\tau_{e} \equiv T_{e} / T_{i}$. From the Ampère's law $k_{\perp}^{2} A_{\|}$ $=4 \pi \delta J_{\|} / c$, we obtain

$$
\begin{aligned}
& \frac{\partial}{\partial k}\left(\frac{1}{2 \tau_{e}} k_{\perp}^{2} \frac{v_{T i}}{c} A_{\|}(k)\right) \\
& =\frac{\partial}{\partial k}\left(\int _ { - \infty } ^ { + \infty } \frac { d k ^ { \prime } } { \sqrt { 2 \pi } } \left[\left\{K_{21}^{i}\left(k, k^{\prime}\right)+K_{21}^{e}\left(k, k^{\prime}\right)\right\} \phi\left(k^{\prime}\right)\right.\right. \\
& \left.\left.+\left\{K_{22}^{i}\left(k, k^{\prime}\right)+K_{22}^{e}\left(k, k^{\prime}\right)\right\} \frac{v_{T i}}{c} A_{\|}\left(k^{\prime}\right)\right]\right) .
\end{aligned}
$$

When using the Ampère's law to determine the lowest order of $A_{\|}$in the $\left(m_{e} / m_{i}\right)^{1 / 2}$-expansion, there simultaneously appear terms including an unknown low-order part of $A_{\|}$which are not negligible. However, these terms are independent of $k$ so that, in Eq. (24), they are eliminated by taking differential with respect to $k$.

In Eqs. (23) and (24), the integral kernels due to ions are defined by 


$$
\begin{aligned}
{\left[\begin{array}{ll}
K_{11}^{i}\left(k, k^{\prime}\right) & K_{12}^{i}\left(k, k^{\prime}\right) \\
K_{21}^{i}\left(k, k^{\prime}\right) & K_{22}^{i}\left(k, k^{\prime}\right)
\end{array}\right]=} & -i \int_{0}^{+\infty} \omega_{* e} d \tau \frac{\sqrt{2} e^{i \omega \tau}}{\sqrt{a \lambda}(1+a)} e^{-\left(k-k^{\prime}\right)^{2} / 4 \lambda} \Gamma_{0}\left(k_{\perp}, k_{\perp}^{\prime}\right)\left[\frac{\omega}{\omega_{* e}} \tau_{e}+1-\frac{3}{2} \eta_{i}+\frac{\eta_{i}\left(k-k^{\prime}\right)^{2}}{4 a \lambda}\right. \\
& \left.+\frac{2 \eta_{i}}{(1+a)}\left(1-\frac{k_{\perp}^{2}+k_{\perp}^{\prime 2}}{2(1+a) \tau_{e}}+\frac{k_{\perp} k_{\perp}^{\prime}}{(1+a) \tau_{e}} \frac{I_{1}}{I_{0}}\right)\right]\left[\begin{array}{cc}
1 & -\frac{\left(k-k^{\prime}\right)}{2 \sqrt{a \lambda}} \\
\frac{\beta_{i}}{\tau_{e}} \frac{\left(k-k^{\prime}\right)}{2 \sqrt{a \lambda}} & -\frac{\beta_{i}}{\tau_{e}} \frac{\left(k-k^{\prime}\right)^{2}}{4 a \lambda}
\end{array}\right],
\end{aligned}
$$

and those due to electrons are given by

$$
\begin{aligned}
K_{12}^{e}\left(k, k^{\prime}\right)= & i \frac{\sqrt{\pi}}{2 \sqrt{2}} \frac{\sqrt{\tau_{e}} q}{|\hat{s}| \epsilon_{n}}\left(\frac{\omega}{\omega_{* e}}-1\right) \frac{\left(k-k^{\prime}\right)}{\left|k-k^{\prime}\right|}, \\
K_{21}^{e}\left(k, k^{\prime}\right)= & -\frac{\beta_{i}}{\tau_{e}} K_{12}^{e}\left(k, k^{\prime}\right), \\
K_{22}^{e}\left(k, k^{\prime}\right)= & \beta_{i} \frac{\sqrt{\pi}}{4 \sqrt{2}}\left(\frac{q}{\hat{s} \epsilon_{n}}\right)^{2}\left|k-k^{\prime}\right|\left[-\frac{\omega}{\omega_{* e}}\left(\frac{\omega}{\omega_{* e}}-1\right)\right. \\
& +2\left|\hat{s} k_{\theta}\right| \epsilon_{n}\left(\frac{\omega}{\omega_{* e}}-1-\eta_{e}\right) \frac{\left(k-k^{\prime}\right)}{\left|k-k^{\prime}\right|} \\
& \left.\times \int_{\theta^{\prime}}^{\theta} d \theta^{\prime \prime} G_{c}\left(\theta^{\prime \prime}\right)\right] .
\end{aligned}
$$

Here, following Dong et al. ${ }^{10}$ we have used variables defined by

$$
\begin{aligned}
& \tau=R q\left|\theta-\theta^{\prime}\right| /\left|v_{\|}\right|, \\
& \lambda=\frac{\tau^{2}}{\tau_{e} a}\left(\frac{\hat{s}}{q} \epsilon_{n}\right)^{2} \omega_{* e}^{2}, \\
& a=1+\frac{2 i \epsilon_{n} \omega_{* i} \tau}{\left(\theta-\theta^{\prime}\right)} \int_{\theta^{\prime}}^{\theta} d \theta^{\prime \prime} G_{c}\left(\theta^{\prime \prime}\right), \\
& k=\left|\hat{s} k_{\theta}\right|\left(\theta-\theta_{k}\right), \quad k^{\prime}=\left|\hat{s} k_{\theta}\right|\left(\theta^{\prime}-\theta_{k}\right), \\
& \Gamma_{0}\left(k_{\perp}, k_{\perp}^{\prime}\right)=I_{0}\left(\frac{k_{\perp} k_{\perp}^{\prime}}{\tau_{e}(1+a)}\right) \exp \left(-\frac{k_{\perp}^{2}+k_{\perp}^{\prime 2}}{2 \tau_{e}(1+a)}\right), \\
& k_{\perp}^{2}=k_{\theta}^{2}\left[1+\Sigma^{2}(\theta)\right], \quad k_{\perp}^{\prime 2}=k_{\theta}^{2}\left[1+\Sigma^{2}\left(\theta^{\prime}\right)\right] .
\end{aligned}
$$

In Eqs. (23)-(32), the wave numbers $k_{\theta}, k_{\perp}$, and $k$ are normalized by $\rho_{s}^{-1}$ with $\rho_{s} \equiv \sqrt{2 T_{e} / m_{i}} / \Omega_{i}$, and $I_{n}$ is the modified Bessel function of order $n$. The integral parameter $\tau$ in Eqs. (25) and (29) has the opposite sign to that in Dong et al. ${ }^{10}$ Equations (22)-(24) form coupled integral eigenmode equations which determine the dispersion relation and the mode structure of the electromagnetic instabilities in helical systems. Instead of using the second integral eigenmode equation by Dong et al. [see Eq. (13) in Ref. 10], we have employed its derivative with respect to $k$ and the constraint given by Eqs. (24) and (22), respectively, although their eigenmode equations and ours both give the same result for $\theta_{k}=0$ because $A_{\|}$for the most unstable mode is an odd func- tion of $k$ for the unstable mode as shown later. [There seem to be typos in Eqs. (16) and (17) of Dong et al., too.] Also, it is shown in Sec III B that, in the fluid limit, the ideal MHD ballooning mode equation is naturally derived from Eqs. (22)-(24), which indicates the validity of our eigenmode equations.

\section{B. Ideal MHD ballooning mode equation}

Here, we consider the fluid limit of Eqs. (22)-(24), in which $\left|k_{\|} v_{T i} / \omega\right| \sim\left|\left(v_{T i} / R_{0} q \omega\right)(\partial / \partial \theta)\right| \ll 1, k_{\perp}^{2} \rho_{T i}^{2} \ll 1$, and $\left|\omega_{D i} / \omega\right| \ll 1$. It can be shown that, in the fluid limit, the integrals including the ion kernels are approximately written by

$$
\begin{aligned}
& \int_{-\infty}^{+\infty} \frac{d k^{\prime}}{\sqrt{2 \pi}} K_{11}^{i}\left(k, k^{\prime}\right) \phi\left(k^{\prime}\right) \\
& \simeq {\left[\tau_{e}+\frac{\omega_{* e}}{\omega}-\left(1+\left(1+\eta_{i}\right) \frac{\omega_{* e}}{\tau_{e} \omega}\right)\right.} \\
&\left.\times\left(\frac{k_{\perp}^{2}}{2}+2 \epsilon_{n} G_{c} \frac{\omega_{* e}}{\omega}+\frac{\tau_{e} v_{T i}^{2}}{2 R_{0}^{2} q^{2} \omega^{2}} \frac{d^{2}}{d \theta^{2}}\right)\right] \phi, \\
& \int_{-\infty}^{+\infty} \frac{d k^{\prime}}{\sqrt{2 \pi}} K_{12}^{i}\left(k, k^{\prime}\right) A_{\|}\left(k^{\prime}\right) \\
& \simeq i\left(1+\left(1+\eta_{i}\right) \frac{\omega_{* e}}{\tau_{e} \omega}\right) \frac{\tau_{e} v_{T i}}{2 R_{0} q \omega} \frac{d A_{\|}}{d \theta}, \\
& \int_{-\infty}^{+\infty} \frac{d k^{\prime}}{\sqrt{2 \pi}} K_{21}^{i}\left(k, k^{\prime}\right) \phi\left(k^{\prime}\right) \\
& \simeq-i\left(1+\left(1+\eta_{i}\right) \frac{\omega_{* e}}{\tau_{e} \omega}\right) \frac{\beta_{i} v_{T i}}{2 R_{0} q \omega} \frac{d \phi}{d \theta}, \\
& \simeq-\left(1+\left(1+\eta_{i}\right) \frac{\omega_{* e}}{\tau_{e} \omega}\right) \frac{\beta_{i}}{2} A_{\|} . \\
& \int_{-\infty}^{+\infty} \frac{d k^{\prime}}{\sqrt{2 \pi}} K_{22}^{i}\left(k, k^{\prime}\right) A_{\|}\left(k^{\prime}\right) \\
& U(36)
\end{aligned}
$$

Using Eqs. (33)-(36), we can rewrite Eqs. (23) and (24) as ordinary differential equations, 


$$
\begin{aligned}
(1- & \left.\frac{\omega_{* e}}{\omega}\right)\left(\phi-i \frac{R_{0} q \omega}{2 c} \int_{-\infty}^{+\infty} d \theta^{\prime} \frac{\left(\theta-\theta^{\prime}\right)}{\left|\theta-\theta^{\prime}\right|} A_{\|}\left(\theta^{\prime}\right)\right)+\left(1+\left(1+\eta_{i}\right) \frac{\omega_{* e}}{\tau_{e} \omega}\right)\left[\left(\frac{k_{\perp}^{2}}{2}+2 \epsilon_{n} G_{c} \frac{\omega_{* e}}{\omega}\right) \phi\right. \\
& \left.+\frac{\tau_{e} v_{T i}^{2}}{2 R_{0} q \omega^{2}} \frac{d}{d \theta}\left(\frac{1}{R_{0} q} \frac{d \phi}{d \theta}-i \frac{\omega}{c} A_{\|}\right)\right]=0,
\end{aligned}
$$

and

$$
\begin{aligned}
\frac{\partial}{\partial \theta}( & \left.\frac{1}{2 \tau_{e}} k_{\perp}^{2} \frac{v_{T i}}{c} A_{\|}\right)+i\left(1+\left(1+\eta_{i}\right) \frac{\omega_{* e}}{\tau_{e} \omega}\right) \frac{\beta_{i} v_{T i}}{2 \omega} \frac{d}{d \theta}\left(\frac{1}{R_{0} q} \frac{d \phi}{d \theta}-i \frac{\omega}{c} A_{\|}\right)+i \frac{\beta_{i} R_{0} q \omega}{\tau_{e} v_{T i}}\left(1-\frac{\omega_{* e}}{\omega}\right)\left(\phi-i \frac{R_{0} q \omega}{2 c}\right. \\
& \left.\times \int_{-\infty}^{+\infty} d \theta^{\prime} \frac{\left(\theta-\theta^{\prime}\right)}{\left|\theta-\theta^{\prime}\right|} A_{\|}\left(\theta^{\prime}\right)\right)-2 \epsilon_{n} G_{c} \frac{\beta_{i} R_{0} q \omega_{* e}}{\tau_{e} v_{T i}}\left(1-\left(1+\eta_{e}\right) \frac{\omega_{* e}}{\omega}\right) \frac{R_{0} q \omega}{2 c} \int_{-\infty}^{+\infty} d \theta^{\prime} \frac{\left(\theta-\theta^{\prime}\right)}{\left|\theta-\theta^{\prime}\right|} A_{\|}\left(\theta^{\prime}\right)=0,
\end{aligned}
$$

respectively. Multiplying Eq. (38) with $i \tau_{e} v_{T i} /\left(\beta_{i} R_{0} q \omega\right)$ and adding it to Eq. (37), we obtain

$$
\begin{aligned}
(1+ & \left.\left(1+\eta_{i}\right) \frac{\omega_{* e}}{\tau_{e} \omega}\right)\left(\frac{k_{\perp}^{2}}{2}+2 \epsilon_{n} G_{c} \frac{\omega_{* e}}{\omega}\right) \phi \\
& +i \frac{v_{T i}}{\beta_{i} R_{0} q \omega} \frac{d}{d \theta}\left(\frac{1}{2 \tau_{e}} k_{\perp}^{2} \frac{v_{T i}}{c} A_{\|}\right) \\
& -2 i \epsilon_{n} G_{c} \frac{\omega_{* e}}{\omega}\left(1-\left(1+\eta_{e}\right) \frac{\omega_{* e}}{\omega}\right) \frac{R_{0} q \omega}{2 c} \\
& \times \int_{-\infty}^{+\infty} d \theta^{\prime} \frac{\left(\theta-\theta^{\prime}\right)}{\left|\theta-\theta^{\prime}\right|} A_{\|}\left(\theta^{\prime}\right)=0 .
\end{aligned}
$$

For low beta $\beta_{i} \ll\left(k_{\|} v_{T i} / \omega\right)^{2}$, we find from Eq. (39) that $\left|\omega A_{\|} / c\right| \ll|\partial \phi / \partial \theta| /\left(R_{0} q\right)$. Then, in this electrostatic case, terms with $A_{\|}$are neglected in Eq. (37), and we have

$$
\begin{gathered}
\frac{\tau_{e} v_{T i}^{2}}{2 R_{0} q \omega^{2}} \frac{d}{d \theta}\left(\frac{1}{R_{0} q} \frac{d \phi}{d \theta}\right)+\left[\frac{k_{\perp}^{2}}{2}+2 \epsilon_{n} G_{c} \frac{\omega_{* e}}{\omega}\right. \\
\left.+\left(1-\frac{\omega_{* e}}{\omega}\right)\left(1+\left(1+\eta_{i}\right) \frac{\omega_{* e}}{\tau_{e} \omega}\right)^{-1}\right] \phi=0,
\end{gathered}
$$

which gives the dispersion relation in the fluid limit for electrostatic modes such as the slab and toroidal ITG modes and the electron drift wave.

Next, let us consider the electromagnetic high-beta case. It is found from Eq. (40) that, in the electrostatic fluid limit, $\left(1-\omega_{* e} / \omega\right) /\left(1+\left(1+\eta_{i}\right) \omega_{* e} /\left(\tau_{e} \omega\right)\right)$ is as small as terms of first order in $\left(k_{\|} v_{T} / \omega\right)^{2}, k_{\perp}^{2}$, and $\left|\omega_{D} / \omega\right|$. For the electromagnetic case, in which the above electrostatic condition for the eigenfrequency is not satisfied, the lowest-order relation between $\phi$ and $A_{\|}$is obtained from Eq. (37) as

$$
\phi-\psi_{\|} \equiv \phi-i \frac{R_{0} q \omega}{2 c} \int_{-\infty}^{+\infty} d \theta^{\prime} \frac{\left(\theta-\theta^{\prime}\right)}{\left|\theta-\theta^{\prime}\right|} A_{\|}\left(\theta^{\prime}\right)=0,
$$

where $\psi_{\|} \equiv i\left(R_{0} q \omega / 2 c\right) \int_{-\infty}^{+\infty} d \theta^{\prime} A_{\|}\left(\theta^{\prime}\right)\left(\theta-\theta^{\prime}\right) /\left|\theta-\theta^{\prime}\right| \quad$ is defined $\left(\psi_{\|}\right.$should not be confused with the poloidal flux function $\psi$ in Sec. II). Taking the derivative of Eq. (41) with respect to $k$ yields

$$
\frac{1}{R_{0} q} \frac{d\left(\phi-\psi_{\|}\right)}{d \theta}=\frac{1}{R_{0} q} \frac{d \phi}{d \theta}-i \frac{\omega}{c} A_{\|}=0,
$$

which represents zero parallel electric field $E_{\|}=0$ as in the ideal MHD. We also note that, because of the boundary conditions $\lim _{\theta \pm \infty} \phi(\theta)=0$ and $\lim _{\theta \pm \infty} \psi_{\|}(\theta)=0$ derived from Eq. (22), the relation in Eq. (41) is also derived from integrating Eq. (42) without an arbitrary integral constant. Thus, the conditions written in Eqs. (41) and (42) are equivalent to each other. We now use these conditions to eliminate $A_{\|}$in Eq. (39) and obtain

$$
\begin{gathered}
\frac{\partial}{\partial \theta}\left[\left(1+\Sigma^{2}\right) \frac{d \phi}{d \theta}\right]+\left[\frac { R _ { 0 } ^ { 2 } q ^ { 2 } } { v _ { A } ^ { 2 } } ( 1 + \Sigma ^ { 2 } ) \left\{\omega^{2}+\left(1+\eta_{i}\right)\right.\right. \\
\left.\left.\times\left(\omega_{* e} / \tau_{e}\right) \omega\right\}+\alpha G_{c}\right] \phi=0,
\end{gathered}
$$

where we have defined the Alfvén velocity $v_{\mathrm{A}}$ $\equiv B_{0} /\left(4 \pi n_{0} m_{i}\right)^{1 / 2}$ and used Eq. (11) and the normalized pressure gradient parameter $\alpha \equiv-R_{0} q^{2} d \beta / d r$ with $\beta \equiv \beta_{i}$ $+\beta_{e} \equiv 8 \pi n_{0}\left(T_{i}+T_{e}\right) / B_{0}^{2}$. In Eq. (43), $\omega_{* i}\left(1+\eta_{i}\right)$ $\equiv-\left(\omega_{* e} / \tau_{e}\right)\left(1+\eta_{i}\right)$ represents the diamagnetic frequency associated with the ion pressure gradient. If the magnitude of the eigenmode frequency is much larger than this ion diamagnetic frequency, Eq. (43) reduces to

$$
\frac{\partial}{\partial \theta}\left[\left(1+\Sigma^{2}\right) \frac{d \phi}{d \theta}\right]+\left[\frac{R_{0}^{2} q^{2}}{v_{\mathrm{A}}^{2}} \omega^{2}\left(1+\Sigma^{2}\right)+\alpha G_{c}\right] \phi=0,
$$

which coincides with the ideal MHD ballooning mode equation.

So far, we have confirmed how the ideal MHD ballooning mode equation shown in Eq. (44) is derived from the kinetic integral eigenmode equations in the fluid limit. Then, the interchange stability criterion, namely, the Mercier criterion can be derived from examining the asymptotic behavior of the solution to Eq. (44) for the case of $\omega=0$ (see Sec. 10.5.4 in Freidberg ${ }^{17}$ ). When deriving the Mercier criterion for equilibria of helical plasmas described in the previous section, we adopt detailed expressions of $\Sigma$ and $G_{c}$ given by 
Eqs. (10) and (13) with help of Eqs. (8), (14), and (16). The resultant Mercier stability criterion is written as

$$
\begin{aligned}
D_{\mathrm{M}} \equiv & \frac{\alpha}{\hat{s}^{2}}\left[\frac{R_{0}}{2} \Omega_{h}^{\prime}-\frac{\alpha}{2}\left(1+\frac{L\left(\epsilon_{h} / \epsilon_{t}\right)}{M q-L}\right)\right. \\
& \left.+\left(\frac{5}{2} \hat{s}-1\right) \Delta^{\prime}+\left(2 \hat{s}-\hat{s}^{2}+r \hat{s}^{\prime}\right) \frac{\Delta}{r}\right] \\
& <\frac{1}{4} .
\end{aligned}
$$

In Eq. (45), the term proportional to $L /(M q-L)(\ll 1)$ may be ignored as a higher-order term in the stellarator expansion. The well-known stabilization due to the magnetic shear is recognized in the denominator of $D_{\mathrm{M}}$. The normal curvature term $R_{0} \Omega_{h}^{\prime} / 2$ associated with the external helical field is a main source of the interchange instability in the helical system. We find that the term proportional to $\alpha$ $\equiv-R_{0} q^{2} d \beta / d r$ is stabilizing because the Shafranov shift caused by increasing $\alpha$ acts favorably on both normal and geodesic curvatures as seen in the previous section. [It should be noted that the Mercier criterion shown in Eq. (45) contains both normal and geodesic curvature effects.] Also, for typical $q$ profiles in the helical system, we have $2 \hat{s}-\hat{s}^{2}$ $+r \hat{s}^{\prime}<0$ and therefore the term proportional to $\Delta$ leads to more stability for larger $\Delta$. On the other hand, for negative magnetic shear $\hat{s} \equiv(r / q)(d q / d r)<0$, the term $\left(\frac{5}{2} \hat{s}-1\right) \Delta^{\prime}$ becomes positive due to the Shafranov shift $\left(\Delta^{\prime}<0\right)$ and thus causes destabilization, which is because $\hat{s} \Delta^{\prime}>0$ acts unfavorably on both normal and geodesic curvatures [see Eqs. (8), (10), (13), (14), and (16)]. These qualitative properties described by Eq. (45) agree well with results from more accurate numerical calculations of the Mercier criterion based on the three-dimensional MHD equilibria. ${ }^{22}$

\section{NUMERICAL RESULTS}

In this section, the coupled integral eigenmode equations shown in Eqs. (22)-(24) are numerically solved to obtain linear growth rates, real frequencies, and mode structures of electromagnetic microinstabilities in a helical system. Employing procedures by Sugama ${ }^{13}$ for proper analytic continuation of the dispersion relation in the complex frequency plane, our numerical code can calculate both positive and negative growth rates, which is useful for accurately determining the critical condition for the marginal stability.

In order to check the validity of our code, we have done a benchmark test using the Cyclone parameters for the Doublet III-D tokamak (see Fig. 1 in Dimits et al. ${ }^{2}$ ). Then, it was found that, in spite of our neglecting trapped ions, growth rates and real frequencies obtained by our code are in good agreement with those by other kinetic codes within deviation of about five percent.

Following the study of electrostatic ITG modes by Kuroda et al., ${ }^{4}$ we consider a system like the LHD and use $L=2, M=10, q=2, \hat{s}=-1, \theta_{k}=0, \alpha_{0}=0, \eta_{i}=\eta_{e}=3.5$, $\epsilon_{n} \equiv L_{n} / R_{0}=0.3, \tau_{e} \equiv T_{e} / T_{i}=1$, and $\epsilon_{h} / \epsilon_{t}=1$ as standard parameters. Here, $\epsilon_{h} / \epsilon_{t}=1$ corresponds to the magnetic sur- (a)

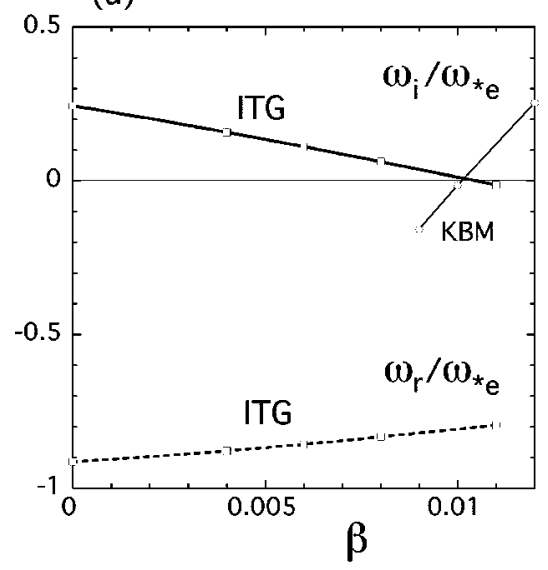

(b)

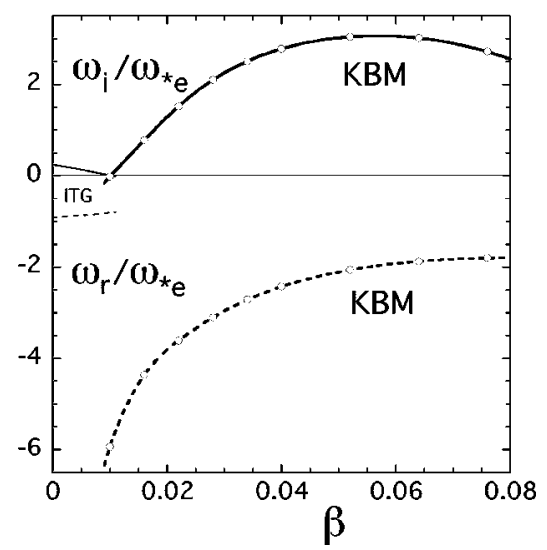

FIG. 1. Normalized growth rate $\omega_{i} / \omega_{* e}$ and real frequency $\omega_{r} / \omega_{* e}$ as a function of $\beta$ for the ITG mode (a) and the KBM (b). Parameters used here are $L=2, M=10, q=2, \hat{s}=-1, \theta_{k}=0, \alpha_{0}=0, \eta_{i}=\eta_{e}=3.5, \epsilon_{n} \equiv L_{n} / R_{0}$ $=0.3, \tau_{e} \equiv T_{e} / T_{i}=1, \epsilon_{h} / \epsilon_{t}=1$, and $k_{\theta} \rho_{T i}=0.5$ (for the ITG mode), 0.35 (for the KBM).

face $r / a \simeq 0.6$. Also, using $\eta_{i}=\eta_{e}=3.5$ and $\epsilon_{n}=0.3$ combined with $R_{0}=3.6 \mathrm{~m}$ and $a=0.6 \mathrm{~m}$ gives the density and temperature gradient scale lengths as $L_{n}=1.08 \mathrm{~m}$ and $L_{T i}$ $=L_{T e}=0.36 \mathrm{~m}$.

Here, we investigate especially how the plasma beta and the magnetic shear affect linear properties of the microinstabilities through geometrical variation of the helical plasma equilibrium. In the LHD, by applying the vertical field, the vacuum magnetic axis is shifted inward in order to realize good particle confinement. In the presence of the pressure gradient, the Shafranov shift works so as to cancel the inward shift by the vertical field. In the present study, we put $\Delta(r)=0$ for simplicity, which represents that, because of the counterbalance between the effects of the vertical field and the pressure gradient, the central axis of the magnetic surface considered coincides with that of the external helical coils. We should note that effects of the toroidal plasma shift due to finite beta are still retained through including $\Delta^{\prime}(r)$ and $\Delta^{\prime \prime}(r)$. The pressure profile inside the flux surface $r$ is necessary in order to evaluate $\Delta^{\prime}(r)$ as shown in Eq. (7) although, in numerical calculations here, we follow Coppi et al. $^{18}$ and Nakajima ${ }^{14}$ to use a simple expression 
(a) ITG $(\beta=0.008)$

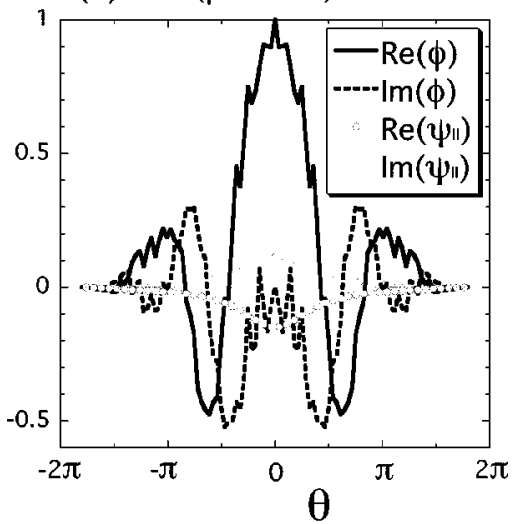

(b) $\mathrm{KBM}(\beta=0.04)$

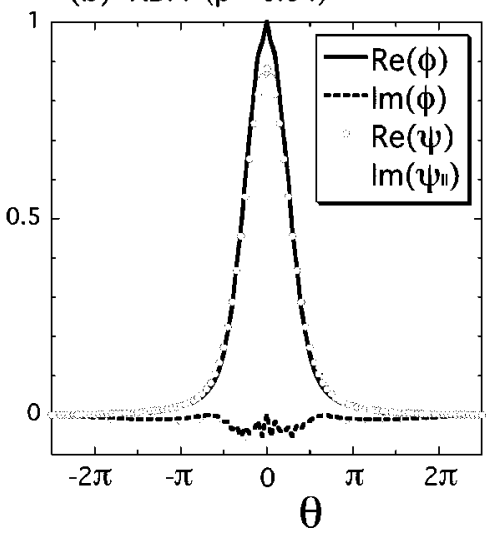

FIG. 2. Eigenfunctions $\phi(\theta)$ and $\psi_{\|}(\theta)$ of the ITG mode for $\beta=0.008$ (a) and of the KBM for $\beta=0.04$ (b). Other parameters used here are the same as in Fig. 1.

$$
\Delta^{\prime}(r)=-\frac{\alpha(r)}{4},
$$

which is a good approximation to Eq. (7) with $\Delta(r)=0$ especially for small $r$. The normalized pressure gradient parameter is rewritten as $\alpha \equiv-R_{0} q^{2} \beta^{\prime}=\left(q^{2} \beta_{i} / \epsilon_{n}\right)\left[\left(1+\eta_{i}\right)\right.$ $\left.+\tau_{e}\left(1+\eta_{e}\right)\right]$.

The normalized real frequency $\omega_{r} / \omega_{* e}$ and growth rate $\omega_{i} / \omega_{* e}$ obtained by numerical calculations using the standard parameters described above are plotted as a function of $\beta$ in Fig. 1. Figures 1(a) and 1(b) correspond to the ITG mode for $k_{\theta} \rho_{T i}=0.5$ and the KBM for $k_{\theta} \rho_{T i}=0.35$, respectively. Here, $k_{\theta} \rho_{T i}=0.5$ and $k_{\theta} \rho_{T i}=0.35$ are the normalized poloidal wave numbers for which the growth rates of the ITG mode and KBM become almost maximum, respectively. Stabilization of the ITG mode and destabilization of the KBM by increasing $\beta$ are seen in the helical system in the same way as observed in the tokamak case ${ }^{10}$ in spite of the opposite sign of the magnetic shear. The eigenfunctions $\phi(\theta)$ and $\psi_{\|}(\theta)$ of the ITG mode for $\beta=0.008$ and the KBM for $\beta=0.04$ are shown in Figs. 2(a) and 2(b), respectively. We see that the ITG mode is essentially electrostatic $(|\phi|$ $\left.\gg\left|\psi_{\|}\right|\right)$while the KBM approximately satisfies $\phi=\psi_{\|}$and accordingly the ideal MHD condition $E_{\|}=0$.

For reference, the averaged normal curvature $R_{0}\langle\Omega\rangle^{\prime} / 2$ and the Mercier parameter $D_{\mathrm{M}}-1 / 4$ are shown as a function of $\beta$ in Figs. 3(a) and 3(b), respectively. Here, we should (a) $s=-1$

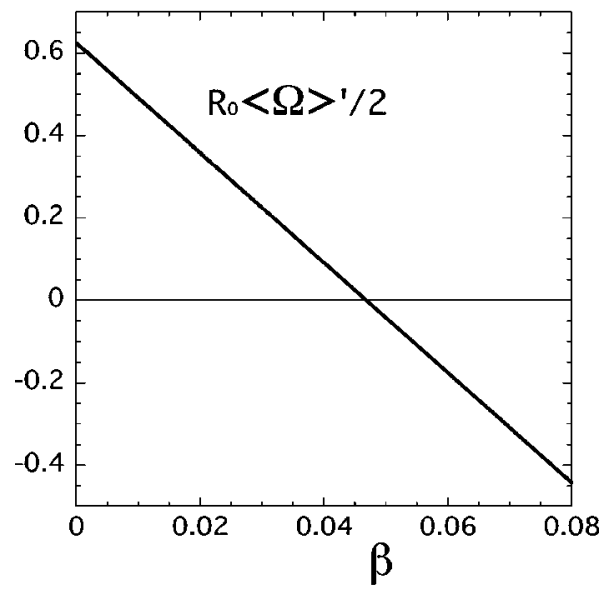

(b) $s=-1$

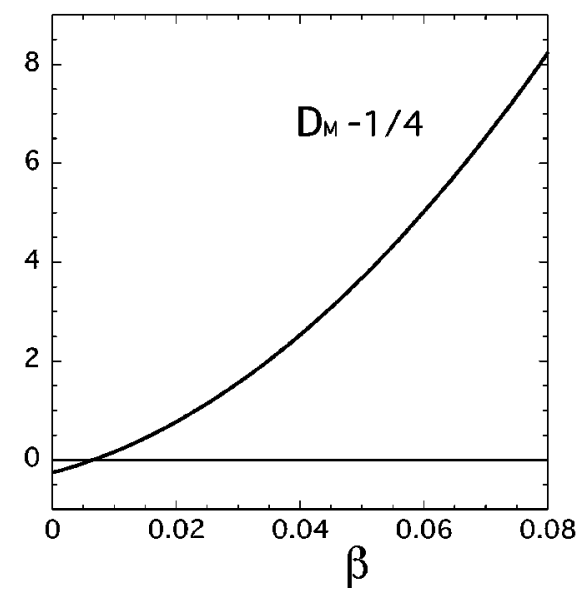

FIG. 3. Averaged normal curvature $R_{0}\langle\Omega\rangle^{\prime} / 2$ (a) and Mercier parameter $D_{\mathrm{M}}-1 / 4$ (b) as a function of $\beta$ for the same parameters as in Fig. 1.

note that, in Figs. 1 and 3, the magnetic configuration is fixed by the standard parameters. In this case, as $\beta$ increases, the averaged normal curvature $R_{0}\langle\Omega\rangle^{\prime} / 2$ monotonically decreases although the Mercier parameter $D_{\mathrm{M}}-1 / 4$, which includes effects of both normal and geodesic curvatures as well as the magnetic shear, monotonically increases. It is confirmed in this helical system that the beta value for the marginal stability of the KBM $(\beta \simeq 0.01)$ is larger than that for the Mercier marginal stability $(\beta \simeq 0.006)$. We also numerically verified that the KBM is completely stabilized for $\beta$ $\geqslant 0.17$ (not shown in Fig. 1) where the helical system is still Mercier-unstable. Thus, in the present case, the KBMunstable region is narrower than the Mercier-unstable region. This is a contrast to the tokamak case that is generally Mercier-stable even when the KBM becomes unstable.

Figure 4 shows that the normalized real frequency $\omega_{r} k_{\theta} \rho_{T i} / \omega_{* e}$ and growth rate $\omega_{i} k_{\theta} \rho_{T i} / \omega_{* e}$ are plotted as a function of the normalized poloidal wave number $k_{\theta} \rho_{T i}$. Here, we employ the $\omega_{* e} /\left(k_{\theta} \rho_{T i}\right)$ as the normalization unit in order to remove the wave number dependence from the unit. Figures 4(a) and 4(b) correspond the ITG mode for $\beta$ $=0$ and the KBM for $\beta=0.02,0.04$, and 0.08 , respectively. It is seen that the KBM can have much larger growth rates 
(a) ITG

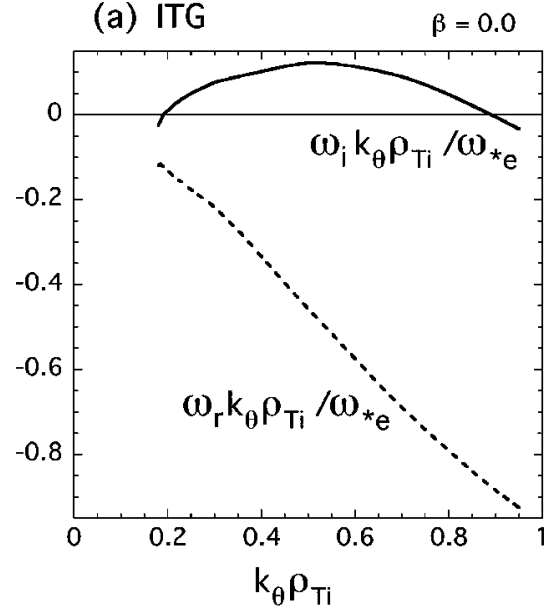

(b) KBM

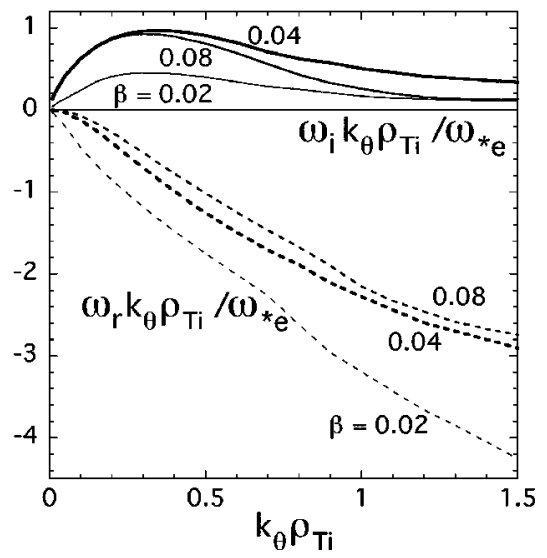

FIG. 4. Normalized growth rate $\omega_{i} k_{\theta} \rho_{T i} / \omega_{* e}$ and real frequency $\omega_{r} k_{\theta} \rho_{T i} / \omega_{* e}$ as a function of $k_{\theta} \rho_{T i}$ for the ITG mode (a) and for the KBM (b). Here, $\beta=0$ for the ITG mode and $\beta=0.01,0.02,0.04,0.08$ for the $\mathrm{KBM}$ are used. Other parameters are the same as in Fig. 1.

and real frequencies and wider unstable wave number regions than those of the ITG. Therefore, the KBM is considered to be a candidate which causes anomalous transport for high beta values.

Figure 5(a) shows the normalized real frequency $\omega_{r} / \omega_{* e}$ and growth rate $\omega_{i} / \omega_{* e}$ of the KBM as a function of the magnetic shear parameter $\hat{s}$ for $k_{\theta} \rho_{T i}=0.35$ and $\beta$ $=0.01,0.02,0.04$, and 0.08. For reference, the averaged normal curvature $R_{0}\langle\Omega\rangle^{\prime} / 2$ and the Mercier parameter $D_{\mathrm{M}}$ $-1 / 4$ are shown as a function of $\hat{s}$ in Figs. 5(b) and 5(c), respectively. As seen in Figs. 5(b) and 5(c), the stronger negative magnetic shear gives the larger normal curvature but it also leads to the Mercier stability for the low-beta case ( $\beta=0.01$ ) by the field-line-bending stabilization of the ideal interchange mode. For the high-beta case $(\beta=0.08)$, the Mercier stability is obtained in the weak magnetic shear region $(\hat{s} \gtrsim-0.3)$, where both normal and geodesic curvatures become favorable. We find from Fig. 5(a) that the stronger magnetic shear brings about stabilization of the electromagnetic microinstability for the lower-beta case, where the mode structure is closer to that of the interchange mode. On the other hand, for higher beta values, the ballooning structure of the kinetic mode becomes more striking and the (a)

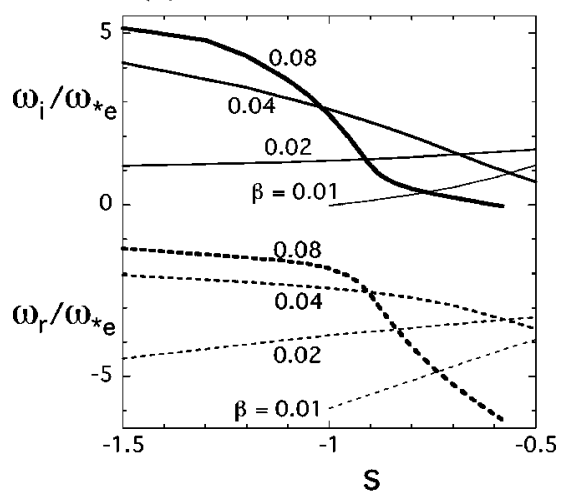

(b)

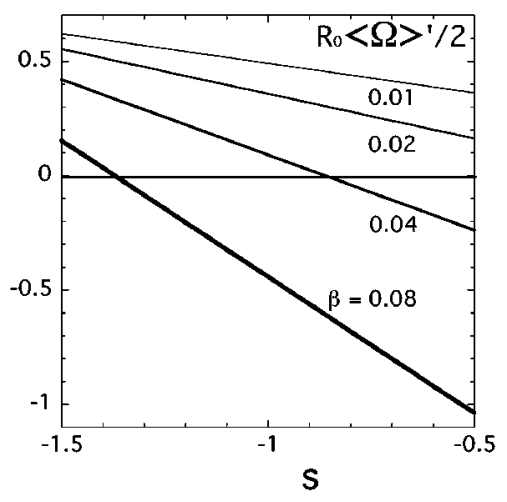

(c)

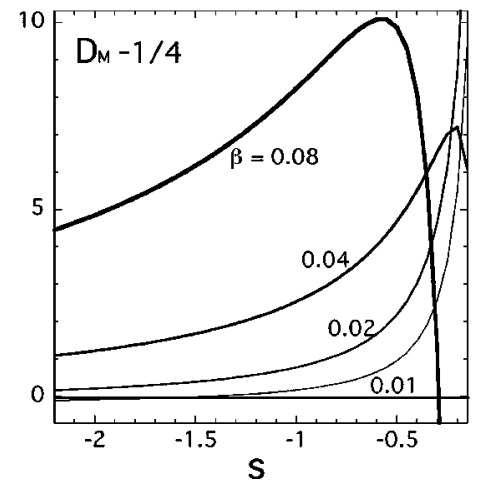

FIG. 5. Normalized real frequency $\omega_{r} / \omega_{* e}$ and growth rate $\omega_{i} / \omega_{* e}$ of the $\mathrm{KBM}$ for $k_{\theta} \rho_{T i}=0.35$ (a), averaged normal curvature $R_{0}\langle\Omega\rangle^{\prime} / 2(\mathrm{~b})$, and Mercier parameter $D_{\mathrm{M}}-1 / 4$ (c) as a function of the magnetic shear parameter $\hat{s}$. Here, $\beta=0.01,0.02,0.04$, and 0.08 are used. Other parameters are the same as in Fig. 1.

stronger (negative) magnetic shear enhances destabilization. Thus, low magnetic shear is expected to be favorable for the high-beta plasma confinement. Comparison between Figs. 5(a) and 5(c) shows some correlation between the stability of the kinetic mode and the Mercier criterion although we find again that the KBM-unstable region is narrower than the Mercier-unstable region.

\section{CONCLUSIONS}

In the present paper, collisionless electromagnetic microinstabilities in helical systems are investigated by using the kinetic integral eigenmode equations derived from the ion 
gyrokinetic equation, the quasineutrality equation, Ampère's law, and the massless electron approximation. Finite-beta effects on the toroidal force balance in the helical systems are taken into account by the stellarator expansion method. The ideal MHD ballooning mode equation is shown to be derived from the kinetic eigenmode equation in the fluid limit. Also, the Mercier criterion obtained from the ideal MHD ballooning equation confirms that, in the helical systems, the negative magnetic shear combined with the Shafranov shift has a destabilizing effect by making both normal and geodesic curvatures unfavorable.

The kinetic integral eigenmode equations are numerically solved to obtain the real frequency, the growth rate, and the mode structure of the electromagnetic microinstability at the core region $(r / a \simeq 0.6)$ in the LHD-like configuration with no net current. Their dependencies on the plasma beta $\beta$, the poloidal wave number $k_{\theta}$, and the magnetic shear parameter $\hat{s}$ are clarified. Stabilization of the ITG mode and destabilization of the KBM by increasing $\beta$ are verified. The $\mathrm{KBM}$ at the high beta $(\beta \geq 0.02)$ has larger growth rates and wider unstable wave number regions than the ITG at the low beta $(\beta \leqq 0.01)$. Therefore, the KBM is expected to cause the anomalous transport in the high-beta case. In the low-beta case $(\beta<0.03)$, the KBM is stabilized by the strong (negative) magnetic shear while, in the high-beta case $(\beta$ $>0.03$ ), it is stabilized by the weak magnetic shear. These tendencies also correlate with the Mercier parameter, which contains effects of the field-line bending and the normal and geodesic curvatures. It is found under the present conditions that the KBM-unstable regions in $\beta$ and $\hat{s}$ are narrower than the Mercier-unstable regions. Thus, kinetic effects such as the Landau damping and the finite gyroradii are considered to play a role of stabilization of the electromagnetic modes so that, for short wavelengths, their stability is better than predicted from the Mercier criterion based on the ideal MHD. This is consistent with the experimental result of the LHD plasma confinement in the Mercier-unstable region.

Since, in the conventional heliotron-type configuration, the stellarator expansion technique describes very well the finite-beta effects on the magnetic hill and on the toroidal plasma shift, which strongly affect stability, the results obtained here are expected to be at least qualitatively valid as well for the case using more complete magnetic geometry given by three-dimensional equilibrium calculations. Also, the single-helicity model of the magnetic field strength used in this paper gives a good approximation to the field strength at $r / a \simeq 0.6$ in the standard LHD configuration for the lowbeta case, where the multiple-helicity effects are small. However, the large magnetic axis shift can be accompanied by increase in the multiple-helicity field components, which in- fluences the distribution of unfavorable curvature regions along the field line. Such multiple-helicity effects are taken into account in the electrostatic microinstability analyses by Kuroda and Sugama ${ }^{23}$ and by Rewoldt et al. ${ }^{5}$ although it seems that there still exist some difficulties in theoretical explanation for the LHD experimental results which show a better confinement for the inward magnetic axis shift with unfavorable magnetic curvature., ${ }^{7,8}$ In addition to the multiple-helicity effects, trapped particles and collisions are not considered in the present electromagnetic analysis. These remain as future problems.

\section{ACKNOWLEDGMENTS}

The authors thank Dr. N. Nakajima, Dr. K. Ichiguchi, Dr. M. Yokoyama, and Dr. Yamagishi for useful discussions on MHD equilibria and stability of helical plasmas.

This work is supported in part by the Japanese Ministry of Education, Culture, Sports, Science, and Technology, Grant Nos. 12680497 and 14780387.

${ }^{1}$ W. Horton, Rev. Mod. Phys. 71, 735 (1999).

${ }^{2}$ A. M. Dimits, G. Bateman, M. A. Beer et al., Phys. Plasmas 7, 969 (2000).

${ }^{3}$ W. M. Tang, J. W. Connor, and R. J. Hastie, Nucl. Fusion 20, 1439 (1980).

${ }^{4}$ T. Kuroda, H. Sugama, R. Kanno, and M. Okamoto, J. Phys. Soc. Jpn. 69, 2485 (2000)

${ }^{5}$ G. Rewoldt, L.-P. Ku, W. M. Tang, H. Sugama, N. Nakajima, K. Y. Watanabe, S. Murakami, H. Yamada, and W. A. Cooper, Nucl. Fusion 42, 1047 (2002)

${ }^{6}$ J. L. V. Lewandowski, Phys. Plasmas 10, 4053 (2003).

${ }^{7}$ O. Motojima, N. Ohyabu, A. Komori et al., Nucl. Fusion 43, 1674 (2003).

${ }^{8}$ H. Yamada, A. Komori, N. Ohyabu et al., Plasma Phys. Controlled Fusion 43, A55 (2001).

${ }^{9}$ O. Yamagishi, Y. Nakamura, K. Kondo, and N. Nakajima, Phys. Plasmas 10, 2871 (2003).

${ }^{10}$ J. Q. Dong, L. Chen, and F. Zonca, Nucl. Fusion 39, 1041 (1999).

${ }^{11}$ T. M. Antonsen, Jr. and B. Lane, Phys. Fluids 23, 1205 (1980).

${ }^{12}$ P. J. Catto, W. M. Tang, and D. E. Baldwin, Plasma Phys. Controlled Fusion 23, 639 (1981).

${ }^{13}$ H. Sugama, Phys. Plasmas 6, 3527 (1999).

${ }^{14}$ N. Nakajima, Phys. Plasmas 3, 4545 (1996).

${ }^{15}$ J. M. Green and J. L. Johnson, Phys. Fluids 4, 875 (1961).

${ }^{16}$ Y. Nakamura, M. Wakatani, and K. Ichiguchi, J. Plasma Fusion Res. 69, 41 (1993).

${ }^{17}$ J. P. Freidberg, Ideal Magnetohydrodynamics (Plenum, New York, 1987), p. 218.

${ }^{18}$ B. Coppi, A. Ferreira, J. W.-K. Mark, and J. J. Ramos, Nucl. Fusion 19, 715 (1979).

${ }^{19}$ R. D. Hazeltine and J. D. Meiss, Plasma Confinement (Addison-Wesley, Redwood City, CA, 1992), p. 298.

${ }^{20}$ R. L. Dewar and A. H. Glasser, Phys. Fluids 26, 3038 (1983).

${ }^{21}$ M. Wakatani, Stellarator and Heliotron Devices (Oxford University Press, Oxford, 1998), p. 98.

${ }^{22}$ K. Ichiguchi, N. Nakajima, M. Okamoto, Y. Nakamura, and M. Wakatani, Nucl. Fusion 33, 481 (1993).

${ }^{23}$ T. Kuroda and H. Sugama, J. Phys. Soc. Jpn. 70, 2235 (2001). 